\title{
PASSIVE ELECTRONIC COMPONETS PARAMETERS SEARCHING WITH DIFFERENTIAL EVOLUTIONARY ALGORITHM
}

This paper describes computing of inductor parameters as passive electronic component with algorithm of differential evolution. Computing of parameters is based on substitute model of inductor which is described with three basic parameters such as resistance, inductance and capacitance. Measured frequency response of complex impedance of the real inductor is input parameter to the iterative loop driven by the differential evolution algorithm. There are three output parameters of the algorithm, where resistance value is considered to be a combination of $D C$ coil resistance and skin effect influence, second value describes inductance of a real inductor and third parameter defines interturn parasitic capacitance of a real inductor.

\section{Introduction}

In the real world one need to find parasitic parameters of real electronic components. This is sometimes a non trivial problem, because of limited measuring equipment capabilities. This paper describes usage of iterative algorithm to compute elements of mathematical model. These components represent parasitic parameters of the real electronic elements. Mentioned iterative algorithm is based on the differential evolution. Mathematical models of basic real electronic components are described in subsequent text.

\section{Equivalent circuit representation of a resistor}

Resistor is a most common circuit element in low frequency electronics, whose purpose is simply to produce a voltage drop by converting some of electric energy into heat. In Fig. 1 is displayed equivalent circuit representation of a resistor.

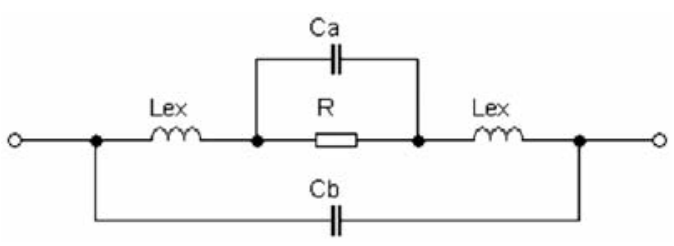

Fig. 1 Electric equivalent circuit representation of a resistor

The interlead capacitance $\mathrm{Cb}$ is usually much smaller than the internal or stray capacitance, and in many cases can be safely neglected. For computing the external inductance of the straight wire at high frequencies can be used formula (1), where $1=2 \mathrm{x}$ (length of single lead) to account for two connections. It should be noted that the preceding formula for the computation of the lead inductance is applicable only for frequencies where the skin depth is smaller than the radius of a wire.

$$
L_{e x}=\frac{\mu_{0} l}{2 \pi}\left[\ln \left(\frac{2 l}{a}\right)-1\right]
$$

Knowing the inductance of the leads, the impedance of the entire circuit can be computed.

$$
Z=j \omega L_{e x}+\frac{1}{j \omega C_{a}+\frac{1}{R}}
$$

The impedance of resistor at low frequencies is equal to the $R$. With increase of frequency effect of the stray capacitance becomes dominant, which causes the impedance of resistor to decrease. Beyond the resonance the total impedance increases due to the lead inductance. Impedance of resistor may be given by formula (2).

\section{Equivalent circuit representation of a capacitor}

Fig. 2 represents equivalent circuit representation of a real capacitor. Impedance of this model of real capacitor is then given by formula (3).

$$
Z_{c}=j \omega L_{e x}+R_{s}+\frac{R_{e}}{R_{e} j \omega C+1}
$$

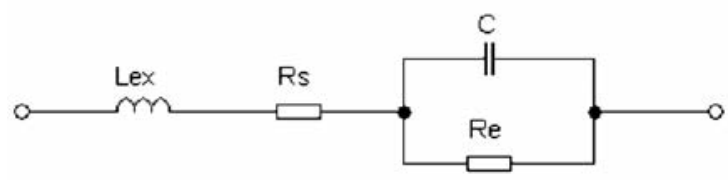

Fig. 2 Electric equivalent circuit representation of a capacitor

\footnotetext{
* Juraj Palecek, Martin Vestenicky

Department of Telecomunications and Multimedia, Faculty of Electrotechnical Engineering, University of Zilina, Slovakia,

E-mail: palecek@fel.uniza.sk
} 
Equivalent circuit representation of an inductor

Although not employed as often as resistors or capacitors, inductors generally are used in transistor biasing networks, for instance as RF chokes (RFCs) to short circuit the device to DC voltage conditions. Since a coil is generally formed by winding a straight wire on a cylindrical former and the windings represent an inductance in addition to the frequency-dependent wire resistance. Moreover, adjacent wires constitute separated moving changes, thus giving rise to a parasitic capacitance effect as shown in Fig. 3.

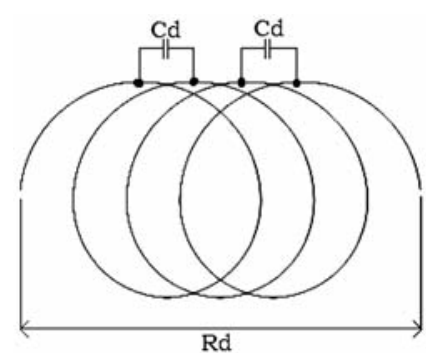

Fig. 3 Distributed capacitance and series resistance in the inductor coil

The equivalent circuit model of the inductor is shown in Fig. 4. The parasitic shunt capacitance Cs and series resistance Rs represents composite effects of capacitance $\mathrm{Cd}$ and resistance $\mathrm{Rd}$ respectively. Impedance of model of real inductor is then given by formula (4).

$$
Z_{L}=\frac{j \omega L+R_{s}}{-\omega^{2} L C_{s}+j \omega R_{s} C_{s}+1}
$$

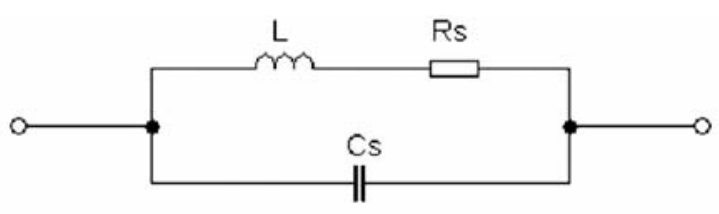

Fig. 4 Electric equivalent circuit representation of an inductor

In the frequency response of the RFC impedance, the impedance increases more rapidly as the frequency approaches resonance. Second, as the frequency continues to increase, the influence of the parasitic capacitance $C S$ becomes dominant and the impedance of the coil decreases. If the RFC has zero series resistance, then the overall impedance behavior at resonance would reach infinity, but due to nonzero contribution of $R s$, the maximum value is finite. To characterize the effect of the coil resistance, the quality factor $Q$ is commonly used. This quality factor characterizes the resistive loss in the passive circuit, and for tuning purposes it is desirable for this factor to be as high as possible. The inductor $Q$ generally increases with frequency, levels off, and then drops close to the self-resonance [3].

\section{Utilized Algorithm and Cost Function Construction}

Differential evolution is a stochastic parallel direct search evolution strategy optimization method that is fairly fast and reasonably robust. Basically, DE adds the weighted difference between two population vectors to a third vector. This way no separate probability distribution has to be used which makes the scheme completely self-organizing [4].

Differential evolution is capable of handling nondifferentiable, nonlinear and multimodal objective functions. It has been used to train neural networks having real and constrained integer weights.

In a population of potential solutions within an n-dimensional search space, a fixed number of vectors are randomly initialized, then evolved over time to explore the search space and to locate the minima of the objective function.

At each iteration called a generation, new vectors are generated by the combination of vectors randomly chosen from the current population (mutation). The outcoming vectors are then mixed with a predetermined target vector. This operation is called recombination and produces the trial vector. Finally, the trial vector is accepted for the next generation if and only if it yields a reduction in the value of the objective function. This last operator is referred to as a selection [5].

The modeling process is based on comparing values of impedance and phase in measured frequencies with values computed with differential evolutionary algorithm. Input parameters of algorithm are values of impedance and angle in measured places of frequency characteristic, and values for algorithm controlling like number of individuals in generation, number of chromosomes of each individual, number of generations, recombination constant and minimum and maximum value chromosome of individual. The output parameter of algorithm is the best individual with the best genes for all of the frequencies in range. That gene is finally used as optimal value for inductor representation.

Each chromosome consists of three genes, where the first gene is used for resistance representation of inductor, second gene describes inductor inductance and third is describing its capacitance.

The genes are represented as decadic numbers. Minimum and maximum values of gene are vectors of least and highest parameters of inductors resistance, inductance and capacity.

Zero generation is generated, where each chromosomes gene is filled with random number from minimum to maximum value of interval at the algorithm start. After that, cost for every chromosome in zero population is computed by "cost" function. Next, the best chromosome with lower cost is considered as "best" chromosome, and its placement is added to new variable. After these steps function of algorithm is stochastic. For defined number of iterations, which is limited by "number of generations" variable, the body of algorithm is processed. There is a randomly generated 
number, which is compared with recombination constant, in every iteration. There is generated a new chromosome from the best chromosome from previous iterations with recombination function, if the value of generated number is greater than recombination constant. Otherwise, the next generation chromosome is created by chromosome from previous iteration. After this, the cost of a new chromosome is computed and if this value is lower than the cost of the best previous chromosome, a new chromosome is justified as the best. If this cost value is higher than the cost of previous chromosome, then this cost is written to the vector of the costs.

Cost value is dedicated as difference between measured and computed frequency response of the impedance and frequency response of the phase in eighteen frequency points. Mathematic formulation of cost value is presented in formula (5).

$$
\begin{aligned}
\text { cost } & =\sum_{i=1}^{18}\left(W_{1}\left|\frac{Z_{\text {computed }}-Z_{\text {measured }(i)}}{Z_{\text {measured }(i)}}\right|+\right. \\
& \left.+W_{2} \mid \frac{\varphi_{\text {computed }}-\varphi_{\text {measured }(i)}}{\varphi_{\text {measured }(i)}}\right)
\end{aligned}
$$

Stop condition of algorithm is bordered with a number of generation variable, where the number of iterations is defined as a real number. In the end of algorithm, the frequency response of impedance (Fig. 6) and frequency response of phase (Fig. 7) is displayed, where values of resistance, conductance and capacity are constituted from the genes of the best chromosome with lower cost. The black points in Figs. 6 and 7 are measured values of impedance and phase of a real inductor. A pseudo-code implementation of algorithm is displayed in Fig. 5. Entire algorithm was realized in Matlab computing language.

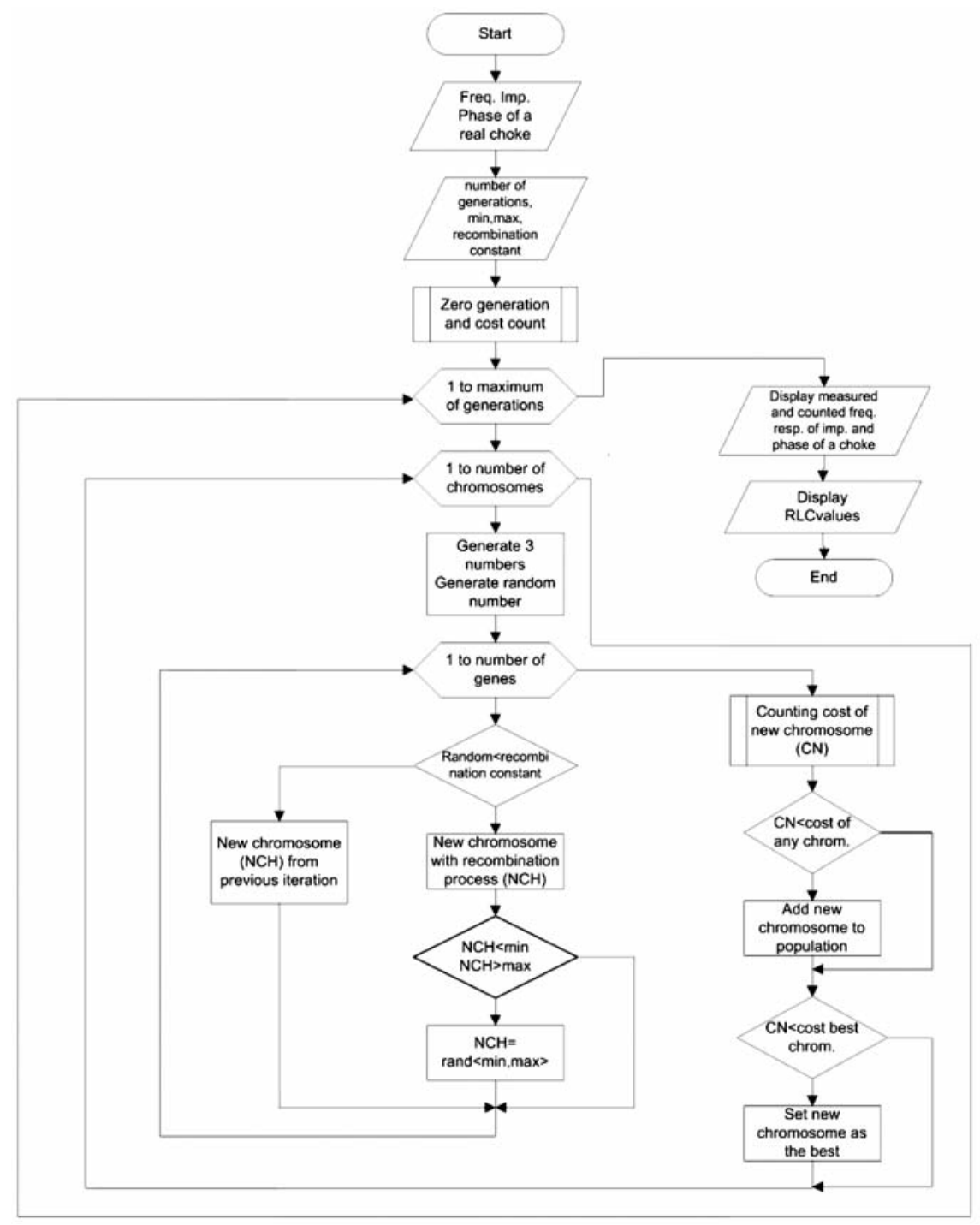

Fig. 5 Pseudo-code implementation of DE algorithm 


\section{Experimental Part}

In this experiment there were computed parameters of a real inductor. This inductor was constructed of $0.5 \mathrm{~mm}$ diameter copper wire with air core. Input parameters such a frequency response of impedance and phase was measured by impedance meter TESLA BM 650 in eighteen frequency steps. Measured frequency range was from $0.5 \mathrm{MHz}$ up to $5 \mathrm{MHz}$. In Table 1 are displayed parameters of differential evolutionary algorithm used in program [1].

Parameters of algorithm

Tab.1

\begin{tabular}{|l|c|}
\hline Parameter & Value \\
\hline Chromosome representation & Decadic \\
\hline Number of unknown coefficients & 3 \\
\hline Differential mutation constant 1 & 0.5 \\
\hline Differential mutation constant 2 & 0.5 \\
\hline Crossing constant & 0.9 \\
\hline Number of chromosomes in generation & 600 \\
\hline Number of generations & 50 \\
\hline Number of genes of chromosome & 3 \\
\hline Calculating time & 33 seconds \\
\hline Calculating time (with skin effect) & 72 seconds \\
\hline W 1 & 1 \\
\hline W 2 & 0.25 \\
\hline
\end{tabular}

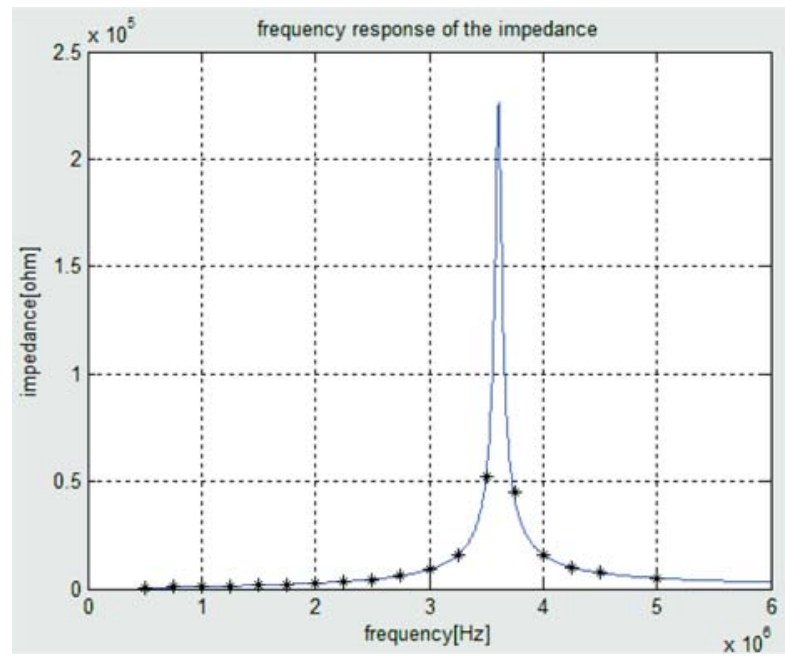

Fig. 6 Frequency response of the impedance of an inductor

\section{Skin effect influence}

Skin effect is a tendency for alternating current to flow mostly near the outer surface of a solid electrical conductor, such as metal wire, at high frequencies. The effect becomes more and more apparent as the frequency increases. The main problem with skin effect is that it increases the effective resistance of a wire for $\mathrm{AC}$ at mod-

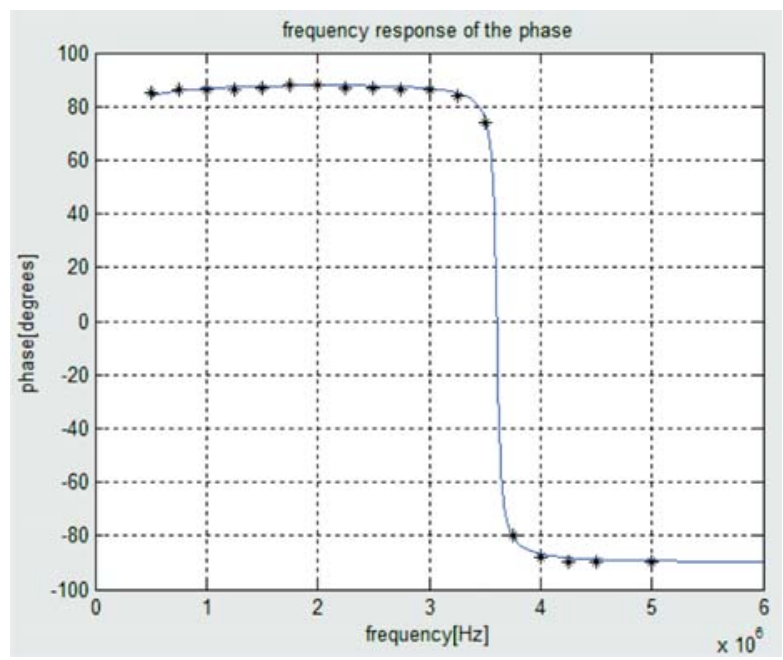

Fig. 7 Frequency response of the phase of an inductor

erate to high frequencies, compared with the resistance of the same wire at direct current and low AC frequencies (Fig. 8). The effect is most pronounced in radio-frequency systems [6].
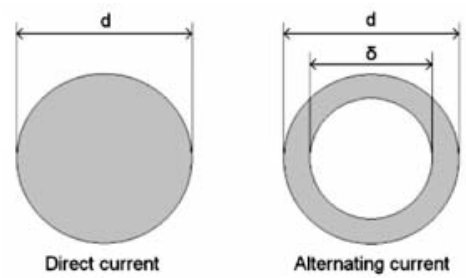

Fig. 8 Skin effect influence

For calculation of skin effect influence formula (6) may be used where $R \sim$ is resistance of inductor with applying skin effect, $R$ is resistance of a wire, $\mathrm{d}$ is diameter and $\delta$ is skin depth (7).

$$
R_{\sim}=R \frac{d}{4 \delta}
$$

The skin depth is defined by formula (7) where material parameters of a wire such specific resistance and permeability of copper are used [4].

$$
\delta=\sqrt{\frac{\rho}{\pi \cdot \mu \cdot f}}
$$

Total resistance of inductor is defined as addition of wire resistance $R_{d c}$ and skin effect resistance $R \sim$.

Frequency response of the impedance and frequency response of the phase with applying skin effect influence are displayed in Figs. 9 and 10. The black points in these figures are measured values of impedance and phase of a real inductor. In Table 2 are displayed results of algorithm like resistance, capacity and inductance without and with applying skin effect influence. 
Results

\begin{tabular}{|l|c|c|}
\hline $\begin{array}{c}\text { Skin effect } \\
\text { influence/component value }\end{array}$ & $\begin{array}{c}\text { Without skin } \\
\text { effect }\end{array}$ & With skin effect \\
\hline Resistance $[\Omega]$ & 48.496 & 49,804 \\
\hline Inductance [mH] & 14.61 & 14.61 \\
\hline Capacity [nF] & 133.15 & 133.15 \\
\hline
\end{tabular}

\section{Conclusion}

The model of inductor with algorithm of differential evolution based on measured values of impedance and phase of frequency

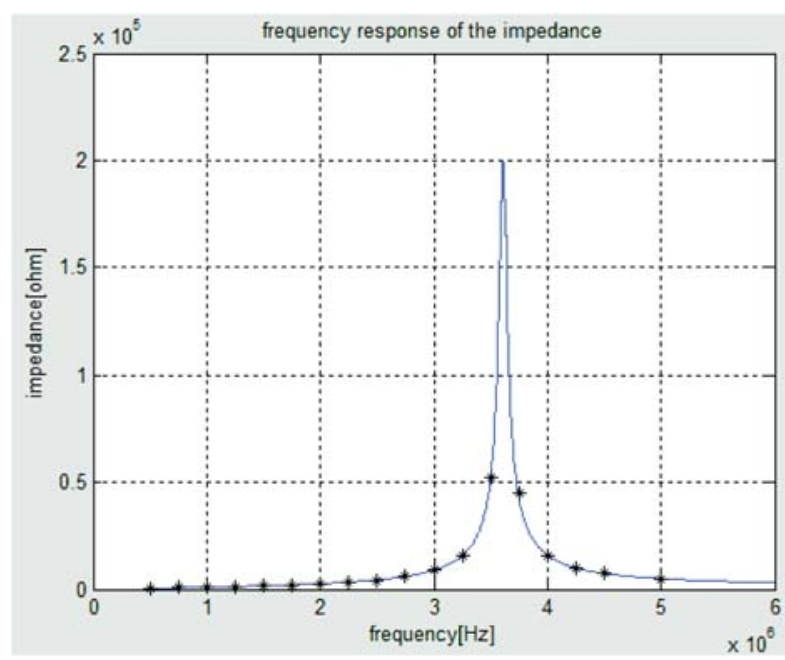

Fig. 9 Frequency response of the impedance of an inductor

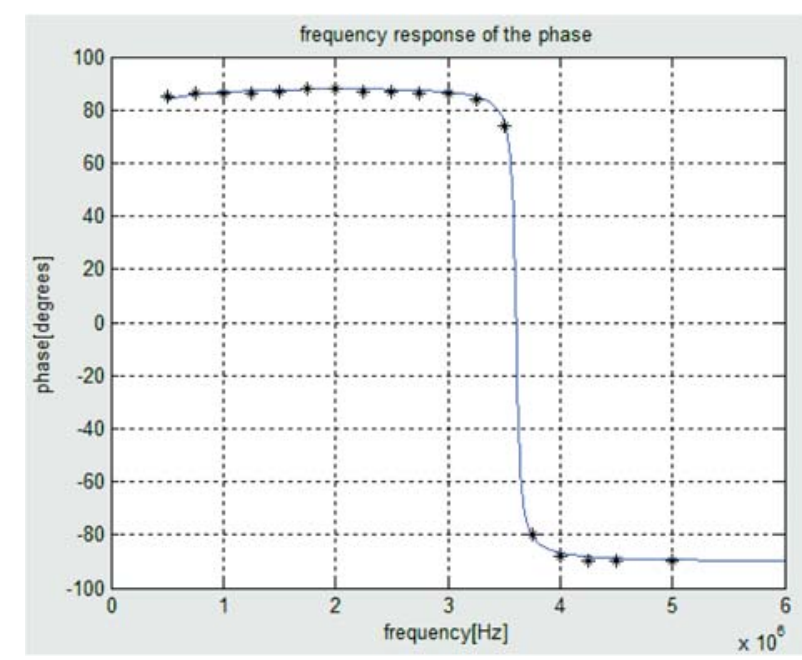

Fig.10 Frequency response of the phase of an inductor with skin effect influence

\section{References}

[1] PAlECEK, J., VESTENICKY, M.: Real Passive Components Parameters Finding With Genetic Algorithm, Proc. of the $8^{\text {th }}$ Int'1 Conference ELEKTRO 2010, Zilina, Slovakia, 2010, ISBN 978-80-554-0196-6.

[2] LUCKA, M., PIECKA, S.: Multi-threaded ant Colony Optimization with Asynchronous Communications for the Vehicle Routing Problem, Communications - Scientific Letters of University of Zilina, Vol. 4, 2010, ISSN 1335-4205.

[3] LUDWIG, R., BOGDANOV G.: RF Circuit Design: Theory and Applications, New Jersey, USA, 2009, ISBN 978-0-13-147137-5.

[3] TICHA, D., MARTINEK, P.: OTA-C Lowpass Design Using Evolutionary Algorithms, Proc. of the 2005 European Conference on Circuit Theory and Design. Cork, University College Cork, 2005, Vol. 2, p. 197-200.

[4] STORN, R.; PRICE, K.: Differential Evolution - a Simple and Efficient Heuristic for Global Optimization over Continuous Spaces, J. of Global Optimization 11, pp. 341-359, 1997.

[5] PRICE, K., STORN, R. M., LAMPINEN, J.A.: Differential Evolution: A Practical Approach to Global Optimization Springer, ISBN 978-3-540-20950-8, 2005.

[6] KUNDERT, K.: Modeling Skin Effect in Inductors, The Designer's Guide, 2006. 